\title{
Effects of sodium-glucose cotransporter 2 inhibitor (dapagliflozin) on food intake and plasma fibroblast growth factor 21 levels in type 2 diabetes patients
}

\author{
Rieko Kosugi ${ }^{1)}$, Eiji Nakatani ${ }^{2), 3)}$, Kensuke Okamoto ${ }^{4)}$, Saeko Aoshima ${ }^{5)}$, Hidekazu Arai ${ }^{4)}$ and \\ Tatsuhide Inoue ${ }^{1)}$ \\ 1) Center for Diabetes, Endocrinology and Metabolism, Shizuoka General Hospital, Shizuoka 420-8527, Japan \\ 2) Division of Statistical Analysis, Research Support Center, Shizuoka General Hospital, Shizuoka 420-8527, Japan \\ 3) Division of Medical Statistics, Translational Research Center for Medical Innovation, Foundation for Biomedical Research and \\ Innovation, Hyogo 650-0047, Japan \\ 4) Division of Laboratory of Clinical Nutrition and Management, School of Food and Nutritional Sciences, The University of \\ Shizuoka, Shizuoka 422-8526, Japan \\ 5) Department of Nutrition, Shizuoka General Hospital, Shizuoka 420-8527, Japan
}

\begin{abstract}
The objective of this study was to investigate whether sodium-glucose cotransporter 2 inhibitors (SGLT2i) treatment in patients with type 2 diabetes induced compensatory hyperphagia by reducing fibroblast growth factor 21 (FGF21) secretion. This prospective study was performed in 26 type 2 diabetes patients treated with dapagliflozin (5 mg/day). Hormonal factors associated with glucose metabolism, dietary intakes estimated by brief self-administered diet-history questionnaire (BDHQ), body weight (BW), and body composition were measured at baseline, and 4 and 12 weeks after dapagliflozin. At 12 weeks, HbA1c levels and BW decreased significantly (both $p<0.0001$ ). BMI at baseline was predictive to baseline $\log _{10}$ (FGF21) $(p=0.037)$. This study showed no change in FGF21, but insulin and glucagon levels decreased significantly (both $p<0.05$ ). Although hyperphagia was found in 10 patients $(38.5 \%)$, defining hyperphagia as $>20 \%$ increase in carbohydrate intake, dapagliflozin treatment induced no hyperphagia, when analyzed by all subjects, and there was no significant association between changes in FGF21 levels and carbohydrate intake. On the other hand, a positive correlation between changes in FGF21 levels or carbohydrate intake and BW was observed (both $p<0.005$ ). Taken together, this study demonstrates that the intervention to maintain the reduced levels in FGF21 is beneficial for BW reduction in type 2 diabetes patients treated with SGLT2i.
\end{abstract}

Key words: Sodium-glucose cotransporter 2 inhibitor, Fibroblast growth factor 21, Carbohydrate intake, Body composition

SODIUM-GLUCOSE COTRANSPORTER 2 INHIBITORS (SGLT2i) reduce plasma glucose concentrations independently of insulin secretion by increasing the renal excretion of glucose. In addition, SGLT2i have an effect on body weight (BW) reduction caused by chronic calorie loss. However, using a mathematical model of human energy metabolism, Ferrannini et al. and Polidori et al. observed that weight loss was much less than the estimated energy loss via glucosuria $[1,2]$. This model indicated that the difference between expected and observed weight loss accounted for the increase in energy intake.

Submitted Jan. 10, 2019; Accepted Apr. 8, 2019 as EJ19-0013 Released online in J-STAGE as advance publication May 25, 2019 Correspondence to: Tatsuhide Inoue, Center for Diabetes, Endocrinology and Metabolism, Shizuoka General Hospital, 4-27-1 Kita Ando, Aoi-ku, Shizuoka 420-8527, Japan.

E-mail: tatsuhide-inoue@i.shizuoka-pho.jp
Although the precise mechanism of overeating caused by SGLT2 $\mathrm{i}$ treatment is unknown, it is assumed that the central nervous system is stimulated by humoral factors to compensate for energy excreted as urine sugar, and appetite is increased. One candidate for such humoral factors is fibroblast growth factor 21 (FGF21), produced by the liver, which is an endocrine hormone that regulates energy homeostasis. FGF21 secretion is stimulated by various nutritional conditions, particularly a high carbohydrate diet. FGF21, secreted by the liver, then enters the circulation and signals to paraventricular neurons in the hypothalamus to reduce sugar intake and BW [3]. This function of negative feedback satiety signal by FGF21 may lead to the development of new anti-obesity agents. These observations have inspired a hypothesis that SGLT2i-associated increases in food intake may be mediated by a decrease in FGF21 levels. 


\section{Materials and Methods}

\section{Study design}

This was a 12 -week, single-center, open-label, singlearm observational study conducted at Shizuoka General Hospital. Outpatients with type 2 diabetes were recruited. Enrolled patients received the SGLT2i dapagliflozin, $5 \mathrm{mg}$ once daily, without changing other antidiabetic agents throughout the whole study period, and the dose was not changed from 12 weeks before the start to the end of the study. During this study, patients were encouraged to visit the hospital every month.

\section{Patients}

Eligible patients were aged 30-75 years and under treatment with anti-diabetic agents including injections of insulin and/or glucagon-like peptide-1 receptor agonist (GLP-1RA). None of the participants had previously been treated with SGLT2i. Patients with a history of cardiovascular disease, malignancy, or pregnancy were excluded.

\section{Collected data}

Blood samples for hemoglobin A1c (HbA1c), plasma glucose, glucose metabolism related factors (plasma FGF21, insulin, glucagon, ketone bodies), lipid profile, renal function, and liver function were collected at overnight fasting states. Dietary intakes were estimated by the BDHQ (Brief self-administered Diet-History Questionnaire). BW and body composition were estimated by dual-energy X-ray absorptiometry (Discovery A; Hologic, Inc, MA, USA).

BDHQ and clinical and biochemical parameters were taken at baseline, then 4 weeks and 12 weeks after administration of dapagliflozin. Body composition was measured at baseline and 12 weeks after dapagliflozin.

\section{Brief self-administered diet-history questionnaire}

Data on dietary intakes were obtained using the $\mathrm{BDHQ}$, a 4-page structured questionnaire that contains questions about 58 foods and beverages and allows mean daily intakes of total energy and 99 different nutrients to be estimated. The food and beverage items listed on the BDHQ were selected to represent foods commonly consumed in Japan, mainly from a food list used in the National Health and Nutrition Survey of Japan. The BDHQ has information on habitual intake, dietary patterns, cooking, and seasoning [4], and is thus used to assess dietary intakes in Japanese adults.

\section{Plasma analyses}

Plasma FGF21 concentrations were measured by ELISA (FGF21 Quantikine ELISA kit; R\&D Systems,
Minneapolis, MN, USA). Plasma ketone bodies were measured by enzymatic cycling method (3-HB-L test kit and TKB-L test kit; Kainos, Tokyo, Japan). Plasma glucagon concentrations were measured by ELISA (Glucagon ELISA kit; Mercodia, Uppsala, Sweden).

\section{Statistical analysis}

Continuous and categorical variables were summarized by means \pm standard deviation (SD) and frequency (percentage), respectively. The differences between clinical parameters at baseline and 4 or 12 weeks were tested using the Wilcoxon signed-rank test. To identify the variables related to $\log$ (FGF21) at baseline and changes in FGF21, we performed linear regression model analysis and determined the slope, $95 \%$ confidence interval, and $p$ value (by Wald test). $P$ values $<0.05$ were considered significant. Statistical analyses were performed using SAS version 9.4 (SAS Institute, Cary, NC, USA).

\section{Ethics}

The study was designed according to the principles stated in the Declaration of Helsinki. The study protocol was approved by the ethics committee of Shizuoka General Hospital (\#15-05-10) and registered on the University Hospital Medical Information Network in Japan (UMIN000013827). All subjects gave written informed consent.

\section{Results}

\section{Patients' characteristics}

Of 35 patients screened, 30 were enrolled in this study. Of these 30 patients, 4 were excluded for reasons of missing values in fasted states and withdrawal of dapagliflozin. Ultimately 26 patients were analyzed.

Table 1 shows the patients' characteristics at baseline. The study included 16 male and 10 female participants. The parameters measured were age, $60.6 \pm 9.1$ years; body mass index (BMI), $26.0 \pm 3.8 \mathrm{~kg} / \mathrm{m}^{2}$; energy intake, $1,593.6 \pm 597.8 \mathrm{kcal}$; and duration of diabetes, $16.8 \pm 9.2$ years. Energy intake estimated by BDHQ was lower by $58.5 \mathrm{kcal}$ than energy intake instructed by medical doctors. At baseline, patients were being treated most frequently with metformin (73.1\%), followed by dipeptidyl dipeptidase-4 (DPP-4) inhibitor (69.2\%), sulfonylurea (30.8\%), insulin (15.4\%), $\alpha$-glucosidase inhibitor (15.4\%), GLP-1RA (7.7\%), and glinide (3.8\%).

\section{Change in parameters}

Changes in biochemical parameters, nutritional states, and body composition between baseline and at 4 and 12 weeks are shown in Table 2. At 12 weeks, HbAlc levels and BW decreased from $8.1 \pm 0.6 \%$ to $7.4 \pm 0.6 \%$, and 
Table 1 Baseline patient characteristics

\begin{tabular}{lc}
\hline variables & statistics \\
\hline Sex (male/female) & $(16 / 10)$ \\
Age (yr) & $60.6 \pm 9.1$ \\
Duration of diabetes (yr) & $16.8 \pm 9.2$ \\
BMI $\left(\mathrm{kg} / \mathrm{m}^{2}\right)$ & $26.0 \pm 3.8$ \\
Energy intake (kcal/day) & $1,593.6 \pm 597.8$ \\
Medication & \\
Insulin (\%) & $4(15.4)$ \\
GLP-1RA (\%) & $2(7.7)$ \\
Sulfonylurea (\%) & $8(30.8)$ \\
Glinide (\%) & $1(3.8)$ \\
Dipeptidyl peptidase 4 inhibitor (\%) & $18(69.2)$ \\
Metformin (\%) & $19(73.1)$ \\
$\alpha$-glucosidase inhibitor (\%) & $4(15.4)$ \\
\hline
\end{tabular}

Continuous variables are expressed as a mean \pm standard deviation. Categorical variables are expressed as number (percentages). BMI, body mass index; GLP-1RA, glucagon-like peptide-1 receptor agonist. from $71.7 \pm 12.6 \mathrm{~kg}$ to $69.6 \pm 11.4 \mathrm{~kg}$ (both $p<0.001$ ), respectively. FGF21 changed from $358.6 \pm 388.7 \mathrm{pg} / \mathrm{mL}$ to $319.7 \pm 312.5 \mathrm{pg} / \mathrm{mL}(p=0.729)$. Insulin and glucagon levels decreased from $11.4 \pm 9.9 \mu \mathrm{IU} / \mathrm{mL}$ to 9.0 $\pm 8.3 \mu \mathrm{IU} / \mathrm{mL}(p=0.049)$ and $57.4 \pm 22.7 \mathrm{pg} / \mathrm{mL}$ to 52.9 $\pm 24.4 \mathrm{pg} / \mathrm{mL}(p=0.031)$, respectively. Ketone bodies significantly increased from $101.7 \pm 70.2 \mu \mathrm{mol} / \mathrm{L}$ to $203.7 \pm 296.4 \mu \mathrm{mol} / \mathrm{L}(p=0.003)$, but with no significant increase in glucagon/insulin ratio $(p=0.312)$. At 4 weeks, changes in FGF21, glucagon levels, and energy intake amounted to $-58.7 \mathrm{pg} / \mathrm{mL},+1.7 \mathrm{pg} / \mathrm{mL}$, and +53.2 kcal, respectively, with no statistical significance.

At 12 weeks, the intake of carbohydrate changed from $212.2 \pm 85.3 \mathrm{~g} /$ day to $212.0 \pm 67.7 \mathrm{~g}$ /day $(p=0.302)$. Hyperphagia was found in 10 patients $(38.5 \%)$, defining hyperphagia as $>20 \%$ increase from baseline in carbohydrate intake.

A positive correlation between changes in carbohydrate intake and BW was observed (regression coefficient: $14.017, p=0.0012$ ).

Table 2 Changes in parameters at baseline and after 4 and 12 weeks of treatment

\begin{tabular}{|c|c|c|c|c|c|}
\hline variables & baseline & 4 weeks & $\begin{array}{c}p \text { value } \\
\text { (baseline } v s .4 \text { weeks) }\end{array}$ & 12 weeks & $\begin{array}{c}p \text { value } \\
\text { (baseline } v s .12 \text { weeks) }\end{array}$ \\
\hline FPG $(\mathrm{mg} / \mathrm{dL})$ & $170.2 \pm 34.8$ & $140.7 \pm 22.2$ & $<0.001$ & $138.0 \pm 23.2$ & $<0.001$ \\
\hline HbAlc (\%) & $8.1 \pm 0.6$ & $7.8 \pm 0.6$ & $<0.001$ & $7.4 \pm 0.6$ & $<0.001$ \\
\hline Insulin $(\mu \mathrm{IU} / \mathrm{mL})$ & $11.4 \pm 9.9$ & $9.9 \pm 9.3$ & 0.051 & $9.0 \pm 8.3$ & 0.0495 \\
\hline Glucagon (pg/mL) & $57.4 \pm 22.7$ & $59.1 \pm 30.6$ & 0.566 & $52.9 \pm 24.4$ & 0.031 \\
\hline Glucagon/Insulin & $1.0 \pm 0.6$ & $1.1 \pm 0.7$ & 0.185 & $1.4 \pm 1.3$ & 0.312 \\
\hline FGF21 (pg/mL) & $358.6 \pm 388.7$ & $299.9 \pm 377.0$ & 0.727 & $319.7 \pm 312.5$ & 0.729 \\
\hline Ketone body $(\mu \mathrm{mol} / \mathrm{L})$ & $101.7 \pm 70.2$ & $167.9 \pm 133.9$ & $<0.001$ & $203.7 \pm 296.4$ & 0.003 \\
\hline $\operatorname{AcAc}(\mu \mathrm{mol} / \mathrm{L})$ & $32.3 \pm 19.7$ & $49.1 \pm 33.3$ & 0.001 & $55.1 \pm 65.7$ & 0.032 \\
\hline 3-OHBA $(\mu \mathrm{mol} / \mathrm{L})$ & $69.4 \pm 50.9$ & $118.8 \pm 104.3$ & 0.005 & $148.5 \pm 235.2$ & 0.044 \\
\hline \multicolumn{6}{|l|}{ Nutritional status } \\
\hline Energy (kcal/day) & $1,593.6 \pm 597.8$ & $1,646.8 \pm 756.7$ & 0.369 & $1,582.1 \pm 495.8$ & 0.425 \\
\hline Carbohydrate (g/day) & $212.2 \pm 85.3$ & $220.9 \pm 99.7$ & 0.192 & $212.0 \pm 67.7$ & 0.302 \\
\hline Protein (g/day) & $62.0 \pm 27.7$ & $67.1 \pm 38.5$ & 0.176 & $61.4 \pm 20.3$ & 0.298 \\
\hline Fat (g/day) & $47.6 \pm 21.6$ & $47.4 \pm 25.8$ & 0.935 & $45.8 \pm 15.7$ & 0.396 \\
\hline \multicolumn{6}{|l|}{ Body composition } \\
\hline Body weight (kg) & $71.7 \pm 12.6$ & & & $69.6 \pm 11.4$ & $<0.001$ \\
\hline Lean body mass (kg) & $52.0 \pm 10.8$ & & & $51.5 \pm 10.2$ & 0.303 \\
\hline Fat mass (kg) & $19.7 \pm 5.4$ & & & $18.0 \pm 5.1$ & $<0.001$ \\
\hline LDL-C (mg/dL) & $114.2 \pm 26.5$ & $114.7 \pm 25.7$ & 0.495 & $120.5 \pm 28.0$ & 0.161 \\
\hline TG (mg/dL) & $126 \pm 50.7$ & $122.5 \pm 41.0$ & 0.521 & $125.7 \pm 48.9$ & 0.379 \\
\hline HDL (mg/dL) & $51.4 \pm 10.2$ & $50.1 \pm 10.6$ & 0.198 & $51.1 \pm 12.5$ & 0.758 \\
\hline ALT (U/L) & $26.9 \pm 10.4$ & $23.1 \pm 10.4$ & 0.006 & $20.2 \pm 7.5$ & 0.001 \\
\hline $\mathrm{eGFR}\left(\mathrm{mL} / \mathrm{min} / 1.73 \mathrm{~m}^{2}\right)$ & $78.3 \pm 20.5$ & $74.9 \pm 23.3$ & 0.021 & $75.6 \pm 23.9$ & 0.096 \\
\hline systolic BP (mmHg) & $133.4 \pm 15.9$ & $125.3 \pm 14.6$ & 0.016 & $125.1 \pm 14.3$ & 0.001 \\
\hline diastolic BP (mmHg) & $78.9 \pm 10.6$ & $76.2 \pm 12.1$ & 0.075 & $74.9 \pm 9.9$ & 0.011 \\
\hline
\end{tabular}

Continuous variables are expressed as a mean \pm standard deviation. FPG, fasting plasma glucose; BMI, body mass index; FGF21, fibroblast growth factor 21; AcAc, acetoacetic acid; 3-OHBA, 3-hydroxybutyric acid. 
Table 3 Correlation between changes in FGF21 and other parameters during this study

\begin{tabular}{lcc}
\hline variables & regression coefficient & $p$ \\
\hline$\Delta$ Carbohydrate intake & 0.283 & 0.205 \\
$\Delta$ Body weight & 12.216 & 0.019 \\
$\Delta$ Fat mass & 0.446 & 0.819 \\
$\Delta$ Lean body mass & 4.949 & 0.186 \\
$\Delta$ Ketone bodies & N/A & N/A \\
$\Delta$ ALT & -0.918 & 0.368 \\
\hline
\end{tabular}

* We treated as "not available (N/A)" due to outliers, although the result was regression coefficient and $p$-value: 0.116 and $p=0.001$.

\section{Correlation between changes in FGF21 and other parameters}

Correlations between changes in FGF21 and other parameters are shown in Table 3. The change in FGF21 levels was not associated with carbohydrate intake, fat mass, lean body mass, and ALT. A positive correlation between changes in FGF21 levels and BW was observed (regression coefficient of BW change for FGF21 change: $12.216, p=0.019)$.

\section{Correlation between FGF21 and BMI at baseline}

FGF21 level at baseline was $358.6 \pm 388.7 \mathrm{pg} / \mathrm{mL}$. $\mathrm{BMI}$ at baseline was predictive to baseline $\log _{10}$ (FGF21) (regression coefficient: $0.038, p=0.037$; Fig. 1), although other parameters were not predictive.

\section{Discussion}

The primary objective of this study was to investigate whether SGLT2i treatment in patients with type 2 diabetes affected FGF21 secretion, inducing compensatory hyperphagia. Although hyperphagia was found in 10 patients (38.5\%), defining hyperphagia as $>20 \%$ increase from baseline in carbohydrate intake, dapagliflozin treatment induced no hyperphagia, when analyzed by all subjects, and there was no significant association between changes in FGF21 levels and carbohydrate intake. On the other hand, a positive correlation between changes in FGF21 levels or carbohydrate intake and BW was observed. Consistent with previous studies, significant decreases in fasting plasma glucose, $\mathrm{HbAlc}$, alanine aminotransferase, blood pressure, BW, and body fat mass, but not in lean body mass, and a significant increase in ketone body were apparent at 12 weeks after dapagliflozin therapy in this study.

Various food intake changes to SGLT2i therapy have been reported in different studies. Compensatory hyperphagia has been reported in several studies of patients with type 1 and type 2 diabetes and also in animals [1, 2,

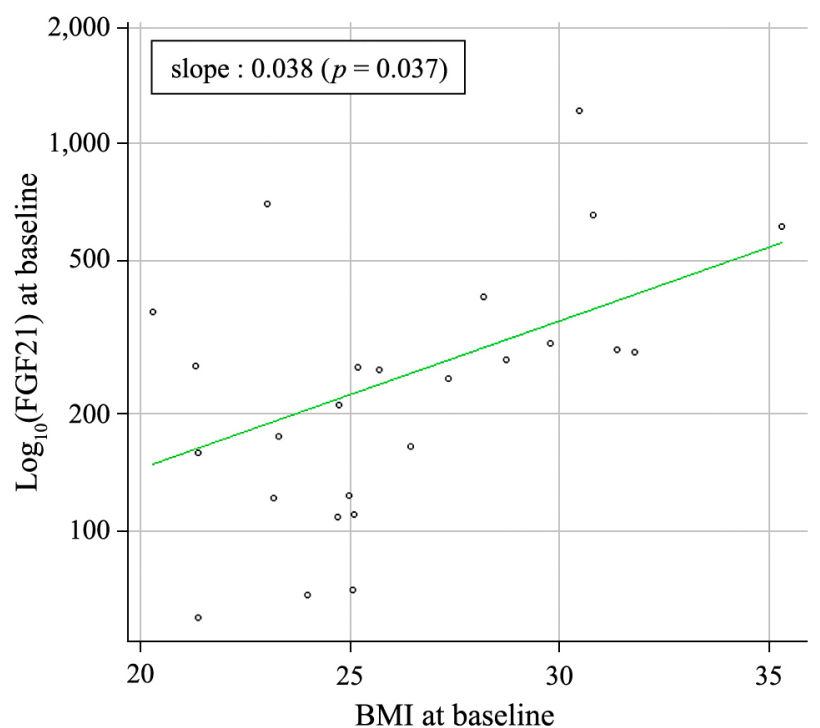

Fig. 1 Correlation between FGF21 and BMI at baseline. FGF21, fibroblast growth factor 21; BMI, body mass index.

$5,6]$. Recently, in contrast to these reports, no change in food and carbohydrate intake after dapagliflozin administration was reported in type 2 diabetes [7], consistent with the results of the present study. In this study, although hyperphagia was found in 10 patients (38.5\%), dapagliflozin treatment induced no hyperphagia, when analyzed by all subjects. It has been shown that the compensatory hyperphagia seen in diabetic mice following dapagliflozin treatment was attenuated by the addition of exenatide [8]. Horie et al. indicated that the daily sucrose intake did not increase in diabetic patients under GLP-1RA treatment after dapagliflozin addition, but increased in patients without GLP-1RA [7]. Ferrannini et $a l$. also showed that the energy intake tended to be lower during SGLT2 inhibition in patients on chronic metformin treatment, who lost significantly more BW than drug-naïve patients [1]. According to these reports, the present results may be explained partly by the use of drugs with anorectic action or by presence of patients who strictly adhere to diet therapy.

Handa et al. reported that plasma levels of FGF21 decreased in the diabetic murine model when fed a lowcarbohydrate diet [9]. Recently, Eriksson et al. indicated that SGLT2i treatment induces a significant reduction in plasma FGF21 levels in comparison with a placebo group [10]. In contrast to these findings, we found no significant decline in FGF21 levels in the present study. It is well known that FGF21 secretion is influenced by several factors such as nutrients, exercise, circadian rhythm, glucagon, insulin, fatty acid, and peroxisome proliferator-activated receptor $\alpha$ [11]. In addition, since SGLT2i treatment influences glucose metabolism, body composition, and secretion of glucagon or insulin, 
among others, SGLT2i treatment could modulate FGF21 secretion in a complex manner, possibly explaining the significant interindividual variation and the lack of decline in FGF21 levels. This explanation may be supported by the recent report that, in pair-fed mice, definitely significant decrease in FGF21 levels after SGLT2i treatment was demonstrated [12].

As lacking FGF21 from the liver demonstrated a preference for high-sucrose diet [3], it is likely that a decrease in FGF21 levels induced by SGLT2i leads to compensatory hyperphagia. Howerver, there was no correlation between changes in FGF21 levels and carbohydrate intake. These findings suggest that acute stimulatory effect of overeating on FGF21 secretion induced no association between reduction of FGF21 levels and increase in carbohydrate intake. Further study is needed to clarify the precise mechanism whereby FGF21 secretion is modulated. In contrast, the positive correlation between changes in carbohydrate intake and BW indicates that the increase in carbohydrate intake secondary to FGF21 reduction suppressed the degree of BW reduction.

Our novel observation was that there was a positive correlation between the change in FGF21 levels and BW over 12 weeks. These findings indicate that changes in the nutritional state following SGLT2 inhibition were involved in a decrease in both FGF21 levels and BW, and that the intervention to maintain the reduced levels of FGF21 is beneficial in type 2 diabetes patients treated with SGLT2i.

We found a positive correlation between FGF21 levels and BMI at baseline in this study. This finding could be supported by the observation that overeating induced an increase in both FGF21 levels and BW, and corresponds to the previous report that plasma FGF21 levels were elevated in obese patients [13].

In this study, the significant reduction in fasting plasma glucagon and insulin levels, but not of the glucagon/ insulin ratio, was observed at 12 weeks after SGLT2i treatment. The acute reaction of glucagon secretion to SGLT2 inhibition was reported to be significantly elevated, which in turn stimulates hepatic glucose production and ketogenesis $[14,15]$. Moreover, even in the chronic phase (12 weeks) of SGLT2i treatment, the fasting glucagon levels remained elevated [14]. The treatment with metformin and/or incretin-related drugs, which lead to suppressed glucagon secretion, was possibly related to the insignificant rise in glucagon levels after dapagliflozin addition in this study [16]. This discrepancy may further be explained by the difference in the glucagon assay method. Recently, Kitamura's group showed that the radioimmunoassay method of glucagon assay is inaccurate compared with the sandwich ELISA method used in this study, which is almost equal in accuracy to liquid chromatography-tandem mass spectrometry [17]. As expected, ketone bodies were significantly elevated at 12 weeks following dapagliflozin addition, but there was no significant association between changes in ketone bodies and the glucagon/insulin ratio. It is possible that large interindividual variation of levels in ketone bodies and glucagon/insulin ratio may account for the lack of this association.

There are several limitations to this study. First, the sample size was small and single arm, implying low statistical power. Second, the correlation between BDHQ and dietary records for energy-adjusted intakes of the 42 nutrients were reported as $0.50-0.56$, meaning that these results must be interpreted with caution. Third, the FGF21 assay used in this study measured total FGF21 and not the bioactive form, which may be the reason for the widely varying baseline FGF21 levels.

In conclusion, this study indicated a positive correlation between changes in FGF21 levels or carbohydrate intake and BW after SGLT2i administration, although no reduction in FGF21 levels nor hyperphagia were observed. Taken together, this study demonstrates that the intervention to maintain the reduced levels in FGF21 is beneficial in type 2 diabetes patients treated with SGLT2i.

\section{Acknowledgments}

The authors thank Tatsuo Ogawa, M.D. (Shizuoka General Hospital) and Yoko Serizawa (dietitian of Shizuoka General Hospital) for their contributions to this trial. This study was funded by the medical research support project of Shizuoka General Hospital.

\section{Disclosure}

None of the authors have any potential conflicts of interest associated with this research.

\section{References}

1. Ferrannini G, Hach T, Crowe S, Sanghvi A, Hall KD, et al. (2015) Energy balance after sodium-glucose cotransporter 2 inhibition. Diabetes Care 38: 1730-1735.
2. Polidori D, Sanghvi A, Seeley RJ, Hall KD (2016) How strongly does appetite counter weight loss? Quantification of the feedback control of human energy intake. Obesity 
(Silver Spring) 24: 2289-2295.

3. von Holstein-Rathlou S, BonDurant LD, Peltekian L, Naber MC, Yin TC, et al. (2016) FGF21 mediates endocrine control of simple sugar intake and sweet taste preference by the liver. Cell Metab 23: 335-343.

4. Kobayashi S, Murakami K, Sasaki S, Okubo H, Hirota N, et al. (2011) Comparison of relative validity of food group intakes estimated by comprehensive and brief-type selfadministered diet history questionnaires against $16 \mathrm{~d}$ dietary records in Japanese adults. Public Health Nutr 14: 1200-1211.

5. Perkins BA, Cherney DZ, Partridge H, Soleymanlou N, Tschirhart H, et al. (2014) Sodium-glucose cotransporter 2 inhibition and glycemic control in type 1 diabetes: results of an 8-week open-label proof-of-concept trial. Diabetes Care 37: 1480-1483.

6. Devenny JJ, Godonis HE, Harvey SJ, Rooney S, Cullen MJ, et al. (2012) Weight loss induced by chronic dapagliflozin treatment is attenuated by compensatory hyperphagia in diet-induced obese (DIO) rats. Obesity (Silver Spring) 20: 1645-1652.

7. Horie I, Abiru N, Hongo R, Nakamura T, Ito A, et al. (2018) Increased sugar intake as a form of compensatory hyperphagia in patients with type 2 diabetes under dapagliflozin treatment. Diabetes Res Clin Pract 135: 178184.

8. Tatarkiewicz K, Polizzi C, Villescaz C, D’Souza LJ, Wang Y, et al. (2014) Combined antidiabetic benefits of exenatide and dapagliflozin in diabetic mice. Diabetes Obes Metab 16: 376-380.

9. Handa $\mathrm{K}$, Inukai $\mathrm{K}$, Onuma $\mathrm{H}$, Kudo A, Nakagawa $\mathrm{F}$, et al. (2014) Long-term low carbohydrate diet leads to deleterious metabolic manifestations in diabetic mice. PLoS One 9: e104948.

10. Eriksson JW, Lundkvist P, Jansson PA, Johansson L,
Kvarnstrom M, et al. (2018) Effects of dapagliflozin and $\mathrm{n}-3$ carboxylic acids on non-alcoholic fatty liver disease in people with type 2 diabetes: a double-blind randomised placebo-controlled study. Diabetologia 61: 1923-1934.

11. Staiger H, Keuper M, Berti L, Hrabe de Angelis M, Haring HU (2017) Fibroblast growth factor 21-metabolic role in mice and men. Endocr Rev 38: 468-488.

12. Ito $\mathrm{S}$, Hosaka $\mathrm{T}$, Yano $\mathrm{W}$, Itou $\mathrm{T}$, Yasumura $\mathrm{M}$, et al. (2018) Metabolic effects of Tofogliflozin are efficiently enhanced with appropriate dietary carbohydrate ratio and are distinct from carbohydrate restriction. Physiol Rep 6: e13642.

13. Zhang X, Yeung DC, Karpisek M, Stejskal D, Zhou ZG, et al. (2018) Serum FGF21 levels are increased in obesity and are independently associated with the metabolic syndrome in humans. Diabetes 57: 1246-1253.

14. Merovci A, Solis-Herrera C, Daniele G, Eldor R, Fiorentino TV, et al. (2014) Dapagliflozin improves muscle insulin sensitivity but enhances endogenous glucose production. J Clin Invest 124: 509-514.

15. Ferrannini E, Muscelli E, Frascerra S, Baldi S, Mari A, et al. (2014) Metabolic response to sodium-glucose cotransporter 2 inhibition in type 2 diabetic patients. $J$ Clin Inves 124: 499-508.

16. Alsalim W, Persson M, Ahren B (2018) Different glucagon effects during DPP-4 inhibition versus SGLT-2 inhibition in metformin-treated type 2 diabetes patients. Diabetes Obes Metab 20: 1652-1658.

17. Miyachi A, Kobayashi M, Mieno E, Goto M, Furusawa K, et al. (2017) Accurate analytical method for human plasma glucagon levels using liquid chromatography-high resolution mass spectrometry: comparison with commercially available immunoassays. Anal Bioanal Chem 409: 5911-5918. 